\title{
Microambiente de la desnutrición marásmica
}

\author{
Margarita Viai R. ${ }^{1}$; Jorge Alvear A. ${ }^{1}$; Carmen Artaza B. ${ }^{1}$ \\ Social backgound in severe undernutrition
}

\begin{abstract}
Undernutrition is associated with precarious sociccultural conditions. Concerned to break the vicious circle of undernutrition and poverty. Chile has developed programs to solve this problem, among these are the Closed Nutritional Recovery Centers (CNAC). where undernourished infants are treated and their families are educa:ed, in order to improve their quality of life and to sustain, after treatment, the nutritional status achieved by their children at the center. We have foltowed up for the past 9 years of 283 children who were admited at age $9.96 \pm 4.97$ imean $\pm \mathrm{SD}$ ) months, (weight/age $63.05 \pm 8.4 \% \mathrm{NCHS}$ standards), for treatment in a CNRC. Only $13 \%$ of them sustained the nutritional status achieved before discharge, which proceeded at age $13.42 \pm 5.1$ months, (weight/age $81.64 \pm 8.9$ ). We also studied, in two transversal points along follow up, thesocio-cultural factors that influence nutrition after discharge from the CNAC: father's alcoholism, the presence of another mal. nourished infant at home, and low socio-economic status at admission were significantly related to bad nutritionat long term evolution, while higher educational level and marital stability favored better outcomes.
\end{abstract}

Key words: undernutrition. sociocultural factors, family.i

La desnutrición calórico-proteica sigue siendo el mayor problema de salud pública para muchos países del tercer mundo. Por regla general, la desnutrición afecta principalmente a niños menores de 6 años, quienes, debido a su acelerada velocidad de crecimiento, tienen requerimientos proporcionalmente muy elevados $y$, además, dependen absolutamente de sus padres u otros adultos para su sustento.

Algunos estudios muestran que más de $40 \%$ de las familias latinoamericanas viven por debajo de lo que se considera nivel crítico de pobreza, lo que se traduce en ura mortalidad infantil 5 veces mayor que la de EE.UU. ${ }^{1,2}$. Este hecho tendría su explicación en la asociación de infección y tasas clevadas de mortalidad, especialmente durante el periodo infantil. Observaciones recientes, epidemiológicas clínicas y de autopsias, sugieren que las deficiencias nutricionales aumentan el riesgo de infección. Entre los factores causantes más corrientes se incluyen la pobreza, manejo sanitario e higiene personal inadecuados, contaminación de agua y comida y educación de slud pública inapropiada ${ }^{1,2}$.

Algunos autores destacan la influencia del ambiente en la génesis del problen nutricional y se

1. INTA. Universidad de Chile. ha descrito que los casos de desnutrición grave aparecen especialmente en familias con microambiente empobrecido e inadecuada relación familiar y que mejorando este ambiente la situación se revierte ${ }^{3,4}$.

En Chile la desnutrición grave sucede de preferencia en lactantes menores que provienen de lugares en malas condiciones habitacionales y alta contaminación ambiental. Rehabilitarlos en el hogar, dados su extrema labilidad y elevado riesgo de infecciones, es diff́cil e incierto. Por estas razones parece importante sacarlos del lugar donde se produjo el daño nutricional, tratándolos en forma eficiente hasta que alcancen un estado que los ponga a resguardo de los riesgos de su ambiente natural. En esta etapa los centros de recuperación nutricional pueden representar mejores soluciones que los hospitales tradicionales por su costo reducido y la posibiljdad de proveer programas de ayuda social que educan e intentan resolver parte de los factores condicionantes de la desnutrición ${ }^{5}$.

En nuestro pais varios programas procuran romper el círculo de desnuttición y pobreza; uno de ellos pertenece a la Corporación para la Nutrición Infantil (CONIN), que ha creado centros cerrados de recuperación nutricional (CCRN) a lo largo del país, donde se trata la desnutrición en un medio adecuado, se educa a las 
madres o personas responsables de los menores y se intenta solucionar los problemas sociales condicionantes, de modo de proporcionar al paciente un ambiente seguro una vez superado el trastorno de la nutrición. El propósito de este estudio fue evaluar la influencia de la familia en la preser. vación del estado de nutrición, obtenido con el tratamiento en estos centros.

\section{Material y Métodos}

Se estudiaron 283 familias de extrema pobreza del área suforiente de Santiago de Chile, cuyos hijos habían ingresado a un CCRN debido a desnutrición severa. La edad promedio de los nifios al ingresar al centro era $8,96 \pm 4,97$ meses y a permanencia en el mismo duró $133,7 \pm 45$ días.

En todos los niños y sus familias se hicieron estudios antropométricos y socioeconómicos. La antropometría incluyó peso, talla, circunferencia de cráneo al ingreso, al alta, en controles quincenales y mensuales, durante un año después del alta y en forma semestral por los 9 años siguientes, siempre con el mismo grupo profesional, que estaba constituido por dos investigadores que realizaron las mediciones en forma independiente, existiendo coincidencia en todos los valores consignados. El peso se determinó con los sujetos desnudos, registrando hasta los $10 \mathrm{~g}$ más próximos. La talla fue medida de pie con talones, naloas $y$ cabeza apoyados firmemente en el antropómetro, hasta el $0,1 \mathrm{~cm}$ próximo. Las circunferencias de brazos $\mathrm{y}$ cabeza se tomaron usando huincha de fibra de vidrio inextensible. La circunferencia de cráneo se tomó considerando los puntos más prominentes de huesos frontales y occipucio hasta el $0,1 \mathrm{~mm}$ próximo. Se realizaron 3 mediciones, obteniéndose el valor promed io. El área grasa y el área magra se detetminaron usando la relación círcunferencia de brazo/pliegue cutáneo según estándares de Frìsancho ${ }^{6}$.

El estado de la nutrición se expresó como porcentaje de peso para la edad ( $\mathrm{P} / \mathrm{E}$ ), talla para la edad ( $\mathrm{T} / \mathrm{E})$, peso para b talla (P/T) y circunferencia craneana para la edad (CC/E) esperados de acuerdo a los estándares OMS. Para el análisis de los resultados se usaron también criterios
OMS, que permiten traducir el ralot obtenido en términos de déficit o exceso oon respecto a la mediana de la población ${ }^{5}$.

La información social fue obtenida mediante entrevista con encuesta estructurada precodificada, a] ingreso, alta y a los 9 años de segujmiento. Ella comprendía la estructura familiar, antecedentes de educación de los padres, características de la vivienda, antecedentes de salud, actividad del menor en estudio, relaclón de pareja, intervención del servicio social y evaluación del programa educativo. El nivel socioeconómico de las familias se describió de acuerdo a una escala de Graffar modificada, que consideró 13 variables con califícación máxima 4 y mínima 0 , mediante la cual la muestra fue dividida en quartiles, originándose 4 niveles, ubicándose el más deficiente en primer lugar ?

El análisis estadístico fue realizado en un computador IBM, usando diferentes pruebas estadísticas (t de Student, chi cuadrado, coeficiente de correlación simple y múltiple), dependiendo del nivel de medición y de las relaciones que se deseaba establecer entre las vartables. Se consideraron diferencias significativas, valores de $\mathrm{p}<0,01$.

\section{Resultados}

La antropometría al ingreso y al alta de los nifios estudiados se resume en la tabla 1 , donde destacan los cambios positivos obtenidos en las relaciones $\mathrm{P} / \mathrm{E}$ y $\mathrm{P} / \mathrm{T}$.

Los indicadores socioeconómicos al ingreso $y$ alta de los niños estudiados no mostraron dife. rencias significativas, por lo que sólo se consideraron los registrados al ingreso y en el corte a los 9 años de seguimiento, para los fines de este estudio. Las familias de los nifros estudiados per. tenecian a extrema pobreza y eran bastante homogéneas en sus características: $22 \%$ de las madres vivían solas y $18,2 \%$ eran analfabetas. Entre los padres $13,3 \%$ eran analfabetos, sólo $24,8 \%$ tenían trabajo estable y $39,5 \%$ sufrian algún grado de alcoholismo. En 50,2\% de las

\section{Tabla I}

Antropometría al ingresar y egresar del centro de recuperación nutricional en 283 pacientes con desnutrición

\begin{tabular}{|c|c|c|c|c|c|}
\hline & \multicolumn{2}{|c|}{ Ingreso } & \multicolumn{3}{|c|}{ Egreso } \\
\hline Edad (meses) & 9,96 & 4,97 & 13,42 & \pm & 5,1 \\
\hline $\mathrm{P} / \mathrm{E}(\%) *$ & 63,05 & $\pm \quad 8,4$ & 81,64 & \pm & 8,9 \\
\hline $\mathbf{T} / \mathbf{E}(\%) *$ & 88,3 & 4,1 & 90,7 & \pm & 3,5 \\
\hline$P / T(\%) *$ & 85,9 & $\pm \quad 8,7$ & 99,8 & \pm & 9,5 \\
\hline
\end{tabular}

* : Estándar OMS: $100 \%$.

P/E : Peso para la edad. T/E: Talla para edad. P/T: Peso para talla. 
familias había otro miembro con antecedentes de desnutrición, situación que bajó a 34\% en el seguimiento, lo que es significativo $(p<0,05)$. Tambiên se redujo el número de padres alcohóli. cos en $10 \%$ y aumentó el número de madres en control de natalidad en igual porcentaje.

La situación socioeconómica desde el ingreso hasta los 9 años de seguimiento (tabla 2) mejoró en el grupo más deteriorado en forma significativa $(\mathrm{p}<0,05)$. Esto se debió fundamentalmente a cambios en la tenencia de la vivienda y las características de ella, ya que los sectores de ex. trema pobreza se beneficiaron con programas habitacionales del gobierno, que mejoraron el saneamiento ambiental y proporcionaron vivien. da a los que no la tenian y vivian como allegados. Sin embargo, cuando tratamos de correlacionar este cambio socioeconómico con la evolución antropométrica no observamos diferencias entre los niños pertenecientes a los 4 grupos de distin. to nivel socioeconómico, por lo que usamos un criterio diferente para analizar la evolución antropométrica, separándo a los niños que habian mantenido o mejorado el estado nutricional del alta de aquellos que se habian deteriorado durante el seguimiento, con to que apareció una relación directa entre la condición socioeconómica al ingresar y la evolución nutricional posterior al alta (tabla 3).

La escolaridad materna, la situación de pareja de los padres, el equipamiento del hogar, especialmente el tipo de combustible empleado para preparar los alimentos, la disposición de excretas y el nivel socioeconómico de ingreso se relacionaron con adecuada evolución nutricional durante el seguimiento (tabla 4).

En la tabla 5 se resumen los factores que aparentemente condicionan la evolución nutricional de este grupo de alto riesgo, observándose que la educación materna y la situación estable

Tabla 2

Nivel socioeconómico al ingresar al centro đe recuperación nutricional y 9 a fó después en 283 familias de pacientes con desnutrición

\begin{tabular}{|c|c|c|c|c|c|c|}
\hline \multirow{2}{*}{ NSE } & \multicolumn{2}{|c|}{ Ingreso } & \multicolumn{2}{|c|}{ Actual } & \multicolumn{2}{|c|}{ Casos estudiados } \\
\hline & $\mathbf{n}$ & $\%$ & $\mathrm{n}$ & $\%$ & n & $\%$ \\
\hline $\begin{array}{l}1 \\
2 \\
3 \\
4\end{array}$ & $\begin{array}{l}86 \\
73 \\
59 \\
65\end{array}$ & $\begin{array}{l}57,7 * \\
49,6 \\
53,1 \\
50,4\end{array}$ & $\begin{array}{l}63 \\
74 \\
52 \\
64\end{array}$ & $\begin{array}{l}42,3 \\
50,3 \\
46,8 \\
49,6\end{array}$ & $\begin{array}{l}149 \\
147 \\
111 \\
129\end{array}$ & $\begin{array}{l}100 \\
100 \\
100 \\
100\end{array}$ \\
\hline & 283 & & 253 & & 536 & \\
\hline
\end{tabular}

$* \quad: \mathrm{p}<0,05$

Tabla 3

Nivel socioeconómico familiar al ingresas al centro de zecuperación nutricional y evolución nutricional según peso pará la edad 9 años después en 283 niños desnutridos

\begin{tabular}{|c|c|c|c|c|c|c|}
\hline \multirow{3}{*}{ NSE } & \multicolumn{4}{|c|}{ Evolución nutricional } & & \\
\hline & \multicolumn{2}{|c|}{ Mala } & \multicolumn{2}{|c|}{ Buena } & \multicolumn{2}{|c|}{ Casos estudiados } \\
\hline & $\mathrm{n}$ & $\%$ & $\mathrm{n}$ & $\%$ & $\mathrm{n}$ & $\%$ \\
\hline $\begin{array}{l}1 \\
2 \\
3 \\
4\end{array}$ & $\begin{array}{l}37 \\
29 \\
26 \\
25\end{array}$ & $\begin{array}{l}61,7 * \\
47,5 \\
43,3 \\
34,7\end{array}$ & $\begin{array}{l}23 \\
32 \\
34 \\
47\end{array}$ & $\begin{array}{l}38,3 \\
52,5 \\
56,6 \\
65,3\end{array}$ & $\begin{array}{l}60 \\
61 \\
61 \\
72\end{array}$ & $\begin{array}{l}100 \\
100 \\
100 \\
100\end{array}$ \\
\hline
\end{tabular}

: $\mathrm{p}<0,05$. 
Tabla 4

Factores relacionados con adecuada evolución nutricional en 283 nirios con desnutrición

\begin{tabular}{|c|c|c|c|c|c|c|}
\hline & \multicolumn{4}{|c|}{ Evolución nutricional } & & \\
\hline & \multicolumn{2}{|c|}{ Mala } & \multicolumn{2}{|c|}{ Buena } & \multicolumn{2}{|c|}{ Casos estudiados } \\
\hline & $\pi$ & $\%$ & $\bar{n}$ & $\%$ & $\mathrm{n}$ & $\overline{q 6}$ \\
\hline \multicolumn{7}{|l|}{ Escolaridad materna: } \\
\hline $\begin{array}{l}\text { Analfabeta } \\
\text { Básica incompleta } \\
\text { Básica completa }\end{array}$ & $\begin{array}{l}22 \\
63 \\
17\end{array}$ & $\begin{array}{l}62,9 * \\
44,4 \\
36,9^{*}\end{array}$ & $\begin{array}{l}13 \\
79 \\
29\end{array}$ & $\begin{array}{l}37,1 \\
55,6 \\
63,1\end{array}$ & $\begin{array}{r}35 \\
142 \\
46\end{array}$ & $\begin{array}{l}100 \\
100 \\
100\end{array}$ \\
\hline \multicolumn{7}{|l|}{ Situación de pareja: } \\
\hline $\begin{array}{l}\text { Madre sola } \\
\text { Madre con pareja }\end{array}$ & $\begin{array}{l}31 \\
85\end{array}$ & $\begin{array}{l}58,5^{*} \\
42,7\end{array}$ & $\begin{array}{r}22 \\
114\end{array}$ & $\begin{array}{l}41,5 \\
57,3\end{array}$ & $\begin{array}{r}53 \\
199\end{array}$ & $\begin{array}{l}100 \\
100\end{array}$ \\
\hline \multicolumn{7}{|c|}{$\begin{array}{l}\text { Tipo de combustible usado } \\
\text { en la cocina: }\end{array}$} \\
\hline $\begin{array}{l}\text { Leña, asertín } \\
\text { Parafina (queto sén) } \\
\text { Gas }\end{array}$ & $\begin{array}{l}14 \\
52 \\
25\end{array}$ & $\begin{array}{l}60,9^{*} \\
38,8 \\
25,3^{*}\end{array}$ & $\begin{array}{r}9 \\
82 \\
74\end{array}$ & $\begin{array}{l}39,1 \\
61,2 \\
74,7\end{array}$ & $\begin{array}{r}23 \\
134 \\
99\end{array}$ & $\begin{array}{l}100 \\
100 \\
100\end{array}$ \\
\hline
\end{tabular}

* : $\mathrm{p}<0,01$.

Tabla 5

Factores socioeconómicos familiares y forma de evolución nutricional en 283 nĩ̛os con desnutrición

\begin{tabular}{llll}
\hline & $\mathbf{P} / \mathrm{E}$ & $\mathrm{T} / \mathrm{E}$ & \\
\hline
\end{tabular}

Evolución buena:

Escolaridad mad̆e adecuada

Situación civil mater na estable

Equipamiento hogar ingreso

Disposicion excretas

Nivel socioeco nómico ingreso

Nivel sociocco nómico actua]

Evolución mala:

Presencia de hermano desnutrido

Alcoholismo del padre

P/T: peso para la edad.

de la pareja parecen favorecerla, mientras que antecedentes de otro integrante de la familia con desnutrición calórico-proteica o alcoholismo paterno aparentemente la perjudican.

\section{Discusión}

La desnutrición en la infancia es un viejo y permanente problema de los países del tercer $\mathbf{p}<0,02$
$\mathbf{p}<0,05$
$\mathbf{p}<0,05$
$\mathbf{p}<0,05$
$\mathbf{p}<0,05$
$\mathbf{p}$

p $<0,02$

$\mathrm{p}<0,01$

$\mathrm{p}<0,01$
$\mathbf{p}<0,05$
$\mathrm{p}<0,05$ 
ma severa que, casi sin excepción, compromete a menores de un año de edad ${ }^{3}$. En Chile la des. nutrición grave ocurre de preferencia en lactantes menores que provienen de hogares de gran precariedad material y alta contaminación, por lo que su rehabjlitación en el hogar, dada su extrema fragilidad, significa aumentar el peligro de infeccion, pareciendo entonces importante alejarlos de los ríesgos propios de su ambiente natural, al menos hasta que alcancen un estado nutricional que los resguarde de ellos.

Parece importante destacar la influencia de los factores socioeconómicos en la nutrición. Una vez más resalta el papel que juega la falta de educación en la generación de problemas nutricionales. Por otra parte, los mejoramientos an la condición socioeconómica tardan en notarse en el bienestar físico de la familia, como parece evidente del hecho que cambios significativos en las condiciones de vivienda y saneamiento ambiental, a lo largo del seguimiento, no se tradujeron en mejoría del estado nutricional de los niños, lo que sugiere que son más importantes los aspectos culturales que los fisicos y que mejores niveles de los primeros permitirian mejor uso de los segundos.

Otro factor que parece importante es la estabilidad de la familia, pues la madre sola o abandonada tiene más dificultad en mantener la situación nutricional de sus hijos.

De la misma manera, en un medio homogéneo de extrema pobreza, donde es difícil discriminar diferencias entre los grupos, un padre alcohólico u otro hermano desnutrido deberían alertar sobre las pocas posibilidades que tiene ese niño de salir adelante en su medio familiar, y obligaría a vigilarlo de cerca, pues si no se observase una respuesta positiva desde el punto de vista nutricional se debería buscar la posibilidad de incorporarlo a una institución o coloca. ción familiar que le garantice mejores condiciones de vida.

\section{Resumen}

La desnutrición se relaciona a malás condiciones socioculturales y hay evidencia que permite establecer clara asociación entre nivel de vida y calidad de la nutrición. En Chile se han formula. do programas para mejorar la situación nutricional de la población infantil. Uno de ellos corresponde al de Centros Cerrados de Recuperación Nutricional. La persistencia de la recuperación obtenida en estas instituciones puede depender en forma importante de la calidad de la situación de la familia. En un seguimiento de 9 años, hecho a niños de extrema pobreza que fueron tratados en uno de tales centros, sólo $13 \%$ conservabar el estado nutricional logrado. Entre los fac. tores que se asocian con evoluciones de sfavorables después del alta destacan el alcoholismo del padre y otro hermano con desnutrición. pero también influyen la educación deficiente y las madres solas.

(Palabras claves: desnutrición, factores sociales, educación, familia.)

\section{Referencias}

1. Altamir, $O$ : La dimensión de la pobreza en Américr Latina, Santiago, Chile: CEPAL, 1978 (L Intorme 180).

2. UNICEF-CEPAL.: Indicadores sobre la situación de la Infancia en América Latina $y$ el Caribe. Santiago, Chile: UNICEF - CEPAL 1979.

3. Winick, M, Meyep, K.K.; Harris, R.C.: Malnutrition and environmental enrichment by early adoption. Science 1975: 190: 1173-1175.

4. My Lien, N.; Meyer, K.K.; Winick, M.: Early malnutrition and late adoption. A study of the effects on development of Korean orphans adopted into American families. Am J Clin Nutr 1977:30: 1743-1749.

5. Monckeberg. F. Centros de Recuperación Nutricional. La experiencia chilena. In: Cxledón, J.M.; ed. Nutrición e inteligencia en el niño. Santiago: Ediciones de la Universidad de Chile, 1983, pp. 39-54.

6. Frisancho, $\boldsymbol{R}$.: New norms of upper limbs fat and muscle arcas for assessment of nutritional status. Am J Clin Nuts 1981, 34: 2540-2545.

7. Alvarez, M.L.; Wurgaft, F.; Wilder, H.: Mediciones del nivel socjoeconómico bajo urbano en familias con kactante desnutrido. Arch Latinoamer Nutr 1982: 32: 650-652. 